\title{
How to Grow a Tree: Plant Voltage-Dependent Cation Channels in the Spotlight of Evolution
}

Ingo Dreyer ${ }^{1, *}$, Frances Sussmilch ${ }^{2,3}$, Kenji Fukushima ${ }^{2}$, Gonzalo Riadi ${ }^{1}$, Dirk Becker ${ }^{2}$, Jörg Schultz ${ }^{4}$, Rainer Hedrich ${ }^{2, *}$

${ }^{1}$ Center for Bioinformatics, Simulation and Modeling (CBSM), Faculty of Engineering, Universidad de Talca, 2 Norte 685, Talca, Chile

${ }^{2}$ Institute for Molecular Plant Physiology and Biophysics, University of Würzburg, Julius-vonSachs-Platz 2, D-97082 Würzburg, Germany

${ }^{3}$ School of Natural Sciences, University of Tasmania, Hobart, TAS 7001, Australia

${ }^{4}$ Department of Bioinformatics, Biozentrum, University of Würzburg, Am Hubland, D-97074

Würzburg, Germany

*Correspondence: idreyer@utalca.cl (I. Dreyer) and hedrich@botanik.uni-wuerzburg.de (R. Hedrich)

Keywords: phylogeny; $\mathrm{K}^{+}$channel; TPC1; plant ion channels

This is the peer-reviewed version of: Dreyer, I. et al. (2020) How to grow a tree: plant voltagedependent cation channels in the spotlight of evolution. Trends Plant Sci, doi: 10.1016/j.tplants.2020.07.011. This article has been published in final form at: https://doi.org/10.1016/j.tplants.2020.07.011.

(C) 2020. This manuscript version is made available under the CC-BY-NC-ND 4.0 license http://creativecommons.org/licenses/by-nc-nd/4.0/ 


\begin{abstract}
Phylogenetic analysis can be a powerful tool for generating hypotheses regarding the evolution of physiological processes. Here, we provide an updated view of the evolution of the main cation channels in plant electrical signalling: the Shaker family of voltage-gated potassium channels and the Two Pore cation $\left(\mathrm{K}^{+}\right)$Channel (TPC1) family. Strikingly, the TPC1 family followed the same conservative evolutionary path as one particular subfamily of Shaker channels $\left(\mathrm{K}_{\text {out }}\right)$ and remained highly invariant after terrestrialisation, suggesting that electrical signalling was, and still remains, key to survival on land. We note that phylogenetic analyses can have their pitfalls, which may lead to erroneous conclusions. To avoid these in the future, we suggest a guideline for analyses of ion channel evolution in plants.
\end{abstract}

\title{
HIGHLIGHTS
}

- New sequence data resources allow the investigation of physiologically important processes in the spotlight of evolution to an unprecedented level of detail.

- Outward-rectifying $\mathrm{K}_{\text {out }}$ channels and TPC1 - cation channels predominantly involved in electrical signalling in angiosperms - remained largely conserved during land plant evolution.

- Inward rectifying potassium channels segregated into different subclades in seed plants, not before their divergence from a common ancestor with ferns.

- Mosses have lost the subclade of outward-rectifying $\mathrm{K}_{\text {out }}$ channels, while lycophytes have lost the inward-rectifying clade of Shaker $\mathrm{K}^{+}$channels.

- Mosses and liverworts (setaphytes) show a second clade of TPC1-type channels, that likely have lost their sensitivity towards cytosolic and luminal $\mathrm{Ca}^{2+}$.

\section{Tracking the evolution of electrical signalling in plants}

Although it is generally accepted that modern land plants evolved from a green algal ancestor, several hypotheses regarding the relative placement of some clades, including bryophytes (mosses, liverworts and hornworts), are still actively discussed. The increased availability of sequence data is enabling more robust analyses as relationships between species in the tree of life are continually revised [1-4]. This data is also driving popularity in research examining the evolution of gene families underlying key traits for major adaptations that likely enabled terrestrialisation. In order 
to thrive on land, plants would have benefitted from signalling pathways to facilitate the transmission of signals between cells in the plant body for coordinated responses to environmental changes. A prerequisite for the evolution of cellular excitability and electrical communication is voltage-gated cation channels that modulate ion fluxes into and out of the cell in response to stressors including drought, heat, and pathogens. The capacity for 'action-potential (AP)'-based electrical signalling, involving changes in cell membrane potential controlled by the sequential activation of ion channels, is also found in some algae and for this reason, these 'green axons' were, in addition to the sensitive Venus flytrap (Dionaea) and Mimosa, among the first model systems used to study the nature of electrical excitability [5-12].

Compared to animals, less is known about the molecular players that drive electrical signalling in plants. While in animals, voltage-dependent sodium channels shape the depolarisation phase of an AP, in plants (Figure 1), it is believed that anion channel-mediated chloride efflux fulfils this role [13]. This depolarization activates voltage-dependent plasma membrane potassium efflux channels, which are currently considered responsible for repolarization of the cell membrane, resetting the cell, ready for the next AP [14,15]. Due to the activity of voltagedependent, $\mathrm{K}^{+}$-permeable channels in the tonoplast, the vacuole membrane also contributes to electrical excitability [16]. The plasma and vacuolar membranes are likely coupled via the $\mathrm{Ca}^{2+}$ wave that accompanies electrical signals (Figure 1; [17]).

Proteins from the plant Shaker family of voltage-dependent plasma membrane $\mathrm{K}^{+}$channels are tetramers built of four subunits, where each subunit comprises six transmembrane domains (S1-S6), a pore-forming loop and helix (P) between S5 and S6 and cytosolic N- and C-termini. In particular the $\mathrm{C}$-terminus contains regulatory sites and the regions important for channel assembly [18-22]. The plant Two-Pore-Channel 1 (TPC1) is a dimer, consisting of two Shaker-like modules linked by a calcium-sensing domain (EF-hands), and encodes a calcium and voltage-dependent cation channel with a main permeability for $\mathrm{K}^{+}[23,24]$ that confers vacuole electrical excitability [16]. Understanding the evolution of electrical signalling in plants requires sequence knowledge of voltage-dependent plant cation channels. The recently published 1KP transcriptomes [3], and genomes of early- and late-diverging streptophyte algae [25-27] and early-diverging land plants $[28,29]$ provide a rich resource from which to mine this information. However, recently published evolutionary analyses highlight the need for awareness of common pitfalls in dealing with large sequence datasets in the context of gene family evolution. 


\section{A Prudent Phylogenetic Approach}

Gene families evolve in complex ways through a variety of events such as gene duplication, deletion, and horizontal gene transfer, and therefore, it is not always easy to accurately reconstruct their phylogenetic relationships. One of the major limitations is the number of nucleotide or amino acid sites available for analysis. The use of coding sequences is often the only option because it is extremely difficult to align the other sequences that comprise or are associated with genes, such as untranslated regions, introns, and flanking intergenic regions, including promoters, especially when distantly related genes or organisms are compared. Therefore, protein sizes, domain architecture and their evolutionary rates are major determinants for the number of phylogenetically informative sites and for the success of phylogenetic analysis. Increased taxon sampling could compensate for such limitations, as it is known to improve the accuracy of tree reconstruction, even with modest amounts of sequences [30]. In addition, there are a few ways to improve phylogenetic trees with further information. Among others, perhaps the most commonly used technique is phylogeny reconciliation, which uses a pre-established species tree (see e.g. Figure S1 in the Online Supplemental Information) to refine less-supported branches based on duplication-loss parsimony [31]. While refining the topology, this method also allows tree rooting and the inference of speciation, duplication, loss, and transfer events, providing a platform for disentangling the complex history of gene family evolution. Large-scale databases such as TimeTree [32] and Open Tree of Life [33] are useful to apply phylogeny reconciliation when species trees are not available otherwise. Gene trees can also be crosschecked with structural and functional information available for the genes/proteins of interest. This step may identify anomalous genes that can bias the analysis, such as nonhomologous genes and genes with a poor quality of annotation. Here we employed the aforementioned methods and provide a comprehensive guide (described in more detail in the Supplementary Information online) for the proper classification of voltage-dependent cation channels in plants using the Shaker and TPC channel families as examples.

\section{Subclasses of Voltage-Gated Plant Potassium Channels}

Pioneering research on voltage-gated plant potassium channels has been carried out on the model plant Arabidopsis thaliana [19]. The Arabidopsis genome encodes nine different subunits of 
voltage-gated potassium channels. According to structural and functional characteristics these were categorized into five subgroups [18,34]: (i) outward-rectifying $\mathrm{K}_{\text {out }}$ channels including SKOR and GORK [35,36], (ii) inward-rectifying $\mathrm{K}_{\mathrm{in}}$-a channels including KAT1 and KAT2 [37-39], (iii) inward-rectifying $\mathrm{K}_{\mathrm{in}} \mathrm{b}$ channels including AKT1, SPIK, and AKT5 [40,41], (iv) weakly rectifying $\mathrm{K}_{\text {weak }}$ channels with the protagonist AKT2 (AKT2/3, AKT3) [42,43], and (v) modulating subunits ( $\mathrm{K}_{\text {silent }}$ AtKC1) that alone do not form functional channels but modulate channel properties in heteromeric assemblies [44-47]. This five-group classification is applicable to all currently known voltage-gated $\mathrm{K}^{+}$channels from angiosperms [48-55]. For gymnosperms and earlier-diverging plants, however, this classification has its limitations (Figure 2A; a fully expanded tree is provided as Figure S2). Channels from these organisms cannot always be assigned to one of these five subgroups because they lack some or all of the characteristic sequence fingerprints that determine the functional differentiation. Instead, it is clear that the five-group distribution of GORK-like, KAT1-like, AKT1-like, AKT2-like and AtKC1-like channels resulted from key evolutionary events (Box 1).

\section{Kout (GORK-like) Channels Were Already Present Before the Divergence of Charophyte Algae and Land Plants}

The origin of plant voltage-gated potassium channels could be traced back to algae [56-58]. Chlorophytes like Volvox, Coccomyxa, Chlamydomonas, Ostreococcus, and Micromonas as well as charophytes including Chlorokybus, Mesostigma, Klebsormidium, Chara, Spirogloea, and Mesotaenium exhibit large sequence variability in putatively voltage-gated potassium channels represented by different clades in phylogenetic trees. With the transition of green plants from an aqueous to a dry environment, this diversity collapsed [53,56]. One structural class (BK-like channels) has been preserved up to gymnosperms, while plant Shaker-like channels are the only voltage-gated $\mathrm{K}^{+}$channels found in angiosperms. The two subclades of this plant Shaker family found already in some late-diverging streptophyte algae including Spirogloea muscicola and Mesotaenium endlicherianum - differ from each other in at least five characteristic structural fingerprints, which also discriminate GORK-like $\mathrm{K}_{\text {out }}$ channels in land plants from the sister branch of $\mathrm{K}_{\mathrm{in}} / \mathrm{K}_{\text {weak }} / \mathrm{K}_{\text {silent }}$ channels (Box 2; Figure 2B; see also Figure $\mathbf{S 3}$ in the Supplemental Information online). Interestingly, these $\mathrm{K}_{\text {out }}$-specific characteristics have not changed significantly during evolution, after their first appearance in streptophyte algae. Some of these 
structural features were shown on the Arabidopsis $\mathrm{K}_{\text {out }}$ channel SKOR to be correlated with particular properties including outward-rectification and the sensing of extracellular $\mathrm{K}^{+}$ concentration [59-64].

An evolutionary particularity of plant Shaker $\mathrm{K}^{+}$channels is that both subbranches, $\mathrm{K}_{\text {in }}$ and $\mathrm{K}_{\text {out, }}$, are present in hornworts, liverworts, ferns, gymnosperms and angiosperms. However, mosses - as represented by the sequenced genomes of Physcomitrella patens [28] and Sphagnum fallax (DOE-JGI) and the transcriptomes of diverse species available from the recent $1 \mathrm{KP}$ project [3] lack the $\mathrm{K}_{\text {out }}$ clade, while lycophytes including Selaginella moellendorffii have lost the $\mathrm{K}_{\text {in }}$ branch [4,56,65]. Although GORK-like channels exist in the late-diverging streptophyte algae $S$. muscicola and M. endlicherianum, the earlier-diverging species Klebsormidium nitens does not possess channels exhibiting the characteristic five-motive $\mathrm{K}_{\text {out }}$-fingerprint (Figure S3). These details refute a recently published opinion [22] which erroneously claimed that GORK-like channels exist in mosses and $K$. nitens based on the misclassification of Shaker channels that lack the hallmarks of GORK-like channels.

\section{Kweak (AKT2-like) Channels Appeared in the Seed Plant Lineage}

$\mathrm{K}_{\text {weak }}$ channels are unique inward-rectifying $\mathrm{K}^{+}$channels which can exist in two different gatingmodes [66-68]: depending on their phosphorylation status, they (1) either activate only at voltages more negative than about $-80 \mathrm{mV}$, and thus allow proton pump-driven $\mathrm{K}^{+}$uptake; or (2) their activation threshold is shifted to far positive voltages with the consequence that the channel is open over the entire physiological voltage-interval. Several characteristic fingerprints discriminate $\mathrm{K}_{\text {weak }}$ channels from the other subclasses (Box 2; Figure 2B). For the Arabidopsis $K_{\text {weak }}$ channel AKT2, the pore region was demonstrated to be responsible for a pronounced block of the channel by $\mathrm{Ca}^{2+}$ ions [42,67,69]. As shown for AKT2 [67,68,70,71] and its rice homolog, OsAKT2 [72], their unique gating $[42,43,73-76]$ could be assigned to a lysine-residue in the S4 voltage-sensor together with two phosphorylation sites in the S4-S5 linker and after the S6 domain. The combined structural fingerprints of $\mathrm{K}_{\text {weak }}$ channels - in particular, the invariable S4 lysine-residue - likely co-occurred with the evolution of seed plants. According to our analysis, and in contrast to another recent report that erroneously misclassified Shaker channel types [76], AKT2-like channels are neither present in algae nor early-diverging land plants. The clade of AKT2-like channels is found only in gymnosperms and angiosperms (Figure 2A; Figure S4; Box 1). 


\section{$K_{\text {silent }}$ (AtKC1-like) Channels are Limited to Angiosperms}

$\mathrm{K}_{\text {silent }}$ channel subunits, like AtKC1 from Arabidopsis and KDC1 from carrot, assemble with other inward-rectifying $\mathrm{K}^{+}$channel subunits to form heteromeric channels with new permeation and gating properties [44-47,77-80]. Although no study has correlated structural motifs with functional properties of these channels, $K_{\text {silent }}$ channels are characterised by distinctive sequence fingerprints (Box 2; Figure 2B; Figure S5). The combination of their structural motifs is found only in angiosperms suggesting that branching of the AtKC1-like clade represents a rather recent event (Figure 2A; Box 1).

\section{Kin-a (KAT1-like) and Kin-b (AKT1-like) Channels Separated with the Appearance of Angiosperms}

Real inward-rectifying channel subunits in Arabidopsis segregate into two subclades: the $\mathrm{K}_{\mathrm{in}}-\mathrm{a}$ clade (KAT1 and KAT2) and the $\mathrm{K}_{\mathrm{in}} \mathrm{-b}$ clade (AKT1, SPIK and AKT6). A remarkable difference between both is that AKT1-like channels require phosphorylation activation by calcium-sensing CBL/CIPK complexes to be competent for sensing voltage changes [77,81-85] while KAT1-like channels do not. From the structural point of view, members of the two clades differ in several characteristic patterns (Box 2; Figure 2B; Figure S6). These distinct patterns are characteristic for $\mathrm{K}^{+}$channels from angiosperms. Inward-rectifying potassium channels from earlier-diverging plant lineages that putatively belong to the $\mathrm{K}_{\text {in }}$ clade show mixtures of these properties and cannot be assigned to either of these two subclades, $K_{i n}-a$ or $K_{i n}-b$. Thus, the separation between KAT1like and AKT1-like channels plausibly occurred during angiosperm evolution, after divergence of gymnosperms (Figure 2A; Box 1).

\section{Two Subclades comprise the Plant Two-Pore-Channel (TPC) family}

TPC channels are found in animal lysosomal compartments and plant vacuoles but seem absent from prokaryotes $[24,86]$. Plant TPC proteins constitute the slow vacuolar cation conductance the SV channel $[23,87]$. Although evolutionarily and structurally related, TPC channels are distinguished from Shaker-like $\mathrm{K}^{+}$channels by consisting of two Shaker domains (D1 and D2) in tandem connected by a cytosolic linker region. Hence, two TPC subunits assemble into a functional ion channel protein $[88,89]$. TPCs from angiosperms have two $\mathrm{Ca}^{2+}$-binding EF-hands 
in their cytosolic linker region [90] and $\mathrm{Ca}^{2+}$-coordination site facing the vacuole lumen [88]. As a consequence, they are gated synergistically by voltage and changes in cytosolic calcium concentrations, while luminal calcium ions affect ion permeation and modulate voltagedependence [91].

Screening of recently available transcriptomes and genomes, and subsequent phylogenetic analysis, revealed that - in contrast to the findings of previous analyses [24,92-94] - the plant TPC family evolved in two directions (Figure 3A; Figure S7). The multiple gene copies that the ancestral algae probably already possessed led to a splitting into the subclade TPC1a (or simply TPC1) encompassing TPC genes from angiosperms, ferns and bryophytes, and the subclade TPC 1b consisting TPC channel variants from mosses and liverworts (setaphytes), only. Automatic domain predictions suggest that TPC $1 \mathrm{~b}$ members seem to almost completely miss the typical EFhand domain architecture in the D1-D2 linker region (Figure S7). A closer examination of this section, however, revealed a pronounced sequence preservation within both subclades, TPC1 and TPC1b (Figure S8). Preliminary structural modelling [95] further proposes that even TPC1b channels still have an EF hand-like conformation in the linker regions. It is not clear though whether these EF-hand-like structures still coordinate $\mathrm{Ca}^{2+}$ ions or whether the differences enable the channels to sense different second messengers.

In contrast to cytosolic $\mathrm{Ca}^{2+}$, which activates angiosperm TPC1, vacuolar (luminal) $\mathrm{Ca}^{2+}$ inhibits channel activity. The amino acid exchange D454N in the Arabidopsis AtTPC1 gain-offunction mutant fou2 (fatty acid oxygenation upregulated 2) renders the SV channel insensitive to inhibitory luminal $\mathrm{Ca}^{2+}[96-98]$. This aspartate residue is part of a luminal $\mathrm{Ca}^{2+}$-coordination site which is highly conserved in members of the TPC 1 subclade, but rather variable in those belonging to TPC 1 b (Figure S9; Figure 3B). Besides the aspartate (D454 in AtTPC1), luminal $\mathrm{Ca}^{2+}$ is further coordinated by side-chain carboxylates from two other residues (e.g. AtTPC1-E528 and AtTPC1D240 from the neighbouring subunit). Most TPC channels from both subclades exhibit the negatively charged side-chains corresponding to D240 in AtTPC1, while at the other two positions one or both negatively charged residues have been replaced with mainly uncharged residues in the TPC1b clade. Depending on the actual pattern of the coordinating motif, this suggests that members of the TPC $1 b$ subclade exhibit impaired luminal $\mathrm{Ca}^{2+}$ sensing. Finally, the observation that the D1 domain in many members of the TPC1b subclade is not properly annotated, is well in line with the finding that this domain evolves faster than the voltage-sensing D2 domain [99]. 


\section{Important Protagonists in Plant Electrical Signalling are Conserved During Land Plant}

Evolution

The evolution of part of the Shaker family and that of the TPC1 family in land plants shows striking similarities. Both, the Shaker $\mathrm{K}_{\text {out }}$ subclade and the TPC1a subclade remained highly invariant after terrestrialisation. This might be a coincidence. However, considering the fact that both channel types are the main cation channels in plant electrical signalling [14,16], it instead suggests that electrical signalling in combination with $\mathrm{Ca}^{2+}$ signalling was, and still is, key to survival on land. In this context, the additional TPC1b clade might compensate for the absence of the $\mathrm{K}_{\text {out }}$ clade in mosses. Although we still do not know the biophysical properties and target membranes

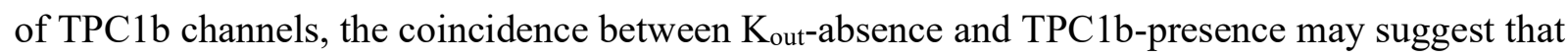
in mosses, members of the TPC1b clade could be targeted to the plasma membrane where they may take over the function of the missing potassium release channels. Alternatively, BK-like potassium channels are also present in mosses and lycophytes $[56,65]$ and might have taken over the tasks of lost Shaker sub-classes. However, plant BK-like channels remain to be functionally characterized. Therefore, it is not yet clear whether they have the biophysical properties to compensate the losses of $\mathrm{K}_{\text {out }}$ and/or $\mathrm{K}_{\text {in }}$ plasma membrane channels.

\section{Pitfalls of Phylogenetic Analyses}

Phylogenetic analysis is not an end in itself but the basis for better understanding of fundamental physiological phenomena in an evolutionary context. To avoid forming distorted views, it is therefore critical to maintain a careful approach and to take all available data into account. This has been exemplified recently in studies that have presented incorrect phylogenetic analyses of voltage-gated plant potassium channels [22,76]. Using Arabidopsis genes as queries, these analyses have been performed with only the highest BLAST match from each considered plant species rather than including all hits. Such an approach, which fails to take into account gene duplication and loss events, misclassified moss $\mathrm{K}_{\text {in }}$ channels as GORK-like channels and lycophyte $\mathrm{K}_{\text {out }}$ channels as AKT2-like channels, for instance [22,76]. As this concerning method has been used as a basic pipeline for numerous gene families [e.g. 100,101], the conclusions drawn in these studies are not placed on proper scientific grounds and should be re-examined. Readers need to be aware of this issue to avoid circulating erroneous interpretations further in the literature. 
Another important issue is the reproducibility of the analyses. For publications of results from wet-laboratory experiments it is indispensable to present all information and material needed to reproduce the experiment. Unfortunately, this good scientific practice has not been followed by several recent bioinformatics studies, which lack the accession details of the analysed genes [e.g. $22,92,102,103]$. Without this essential information it is difficult for peers to detect potential errors in the analyses and, even more problematic, it hinders other researchers from building on the work and directly investigating the genes identified in functional analyses.

The overinterpretation of sequence data is also a potential issue. It should always be taken into account that genetic information in the databases might be incomplete or genes might be improperly annotated (e.g. see Figure $\mathbf{S} 7$ for two C. quinoa TPC1 genes that are annotated with spurious deletions in the latest Phytozome gene models but not in the NCBI Gene database). It is risky to interpret sequence gaps without wet-laboratory experimental confirmation as structural adaptation of an organism to environmental conditions [92], especially if the gaps are in structurally conserved protein domains, coincide with annotated exon boundaries, and/or if additional sequence information is available in the databases that do not show these gaps.

\section{Concluding Remarks and Future Perspectives}

Phylogenetic analyses can be a powerful tool to generate plausible hypotheses for the evolution of physiologically important processes. The growing amount of sequencing data provides a rich resource from which to mine valuable information. Nevertheless, phylogenetic analyses have their pitfalls, as some recent inaccurate publications have shown. Neglecting available information can very quickly lead to erroneous conclusions becoming established as alternative facts.

Examination of the evolution of the voltage-gated plant cation channels shown to be involved in electrical signalling in angiosperms, reveals surprising parallels. Despite the fact that $\mathrm{K}_{\text {out }}$ channels appeared suddenly in streptophyte algae, our picture of the evolution of Shaker $\mathrm{K}^{+}$ channels, which was developed in 2012 [56] with the few data resources available at that time, is still current and has since been further corroborated and refined $[4,53,57,58,65]$. Now, we see that the TPC1 family took the same highly-conservative evolutionary path as the $\mathrm{K}_{\text {out }}$ subfamily. Based on this observation, future studies will need to investigate whether the basis of electrochemical signalling in land plants was already firmly established during the transition from an aqueous to a dry environment (see also Outstanding Questions). 


\section{Acknowledgements}

This work was funded in part by grants of the DFG priority programme 'MAdLand - Molecular Adaptation to Land: plant evolution to change' to RH and DB. KF acknowledges funding by the Sofja Kovalevskaja programme by the Alexander von Humboldt Foundation. FS is supported by an Australian Research Council Discovery Early Career Award (DE200101133) funded by the Australian Government.

\section{OUTSTANDING QUESTIONS}

- Many important structural motifs in $\mathrm{K}_{\text {out }}$ channels appeared suddenly in some latediverging streptophyte algae without a clear evolutionary origin. Were they acquired by horizontal gene transfer?

- Channels of the TPC1b subclade in mosses and liverworts differ in characteristic features from vascular plant TPC1-type channels. Are TPC1b-type channels targeted to the plasma membrane, and do they fulfil different functions and compensate for the missing $\mathrm{K}_{\text {out }}$ channels in mosses?

- $\mathrm{K}_{\text {out }}$ channels and normal TPC1 channels did not change substantially during land plant evolution. Was the basis for electrical signalling in plants already set during the transition from an aqueous to a dry environment?

- What are the functional properties of the Shaker channels from early-diverging streptophyte algae, which lack the characteristic features of channels of land plants?

- How do lycophytes compensate for the loss of the $\mathrm{K}_{\text {in }}$ branch? 


\section{Glossary}

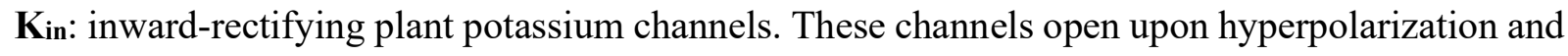
allow the uptake of $\mathrm{K}^{+}$into the cytosol.

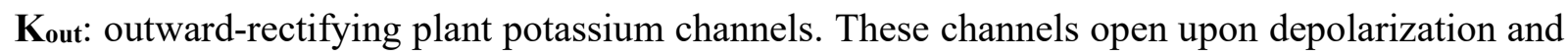
allow the release of $\mathrm{K}^{+}$into the apoplast. Their activity is modulated by the external $\mathrm{K}^{+}$ concentration.

Kweak: weakly-rectifying, bi-modal plant potassium channels. These are specialized $\mathrm{K}_{\text {in }}$ channels that are switched by post-translational modifications between an inward-rectifying mode and an open mode.

$\mathbf{K}_{\text {silent: }}$ silent potassium channel subunits. Together with $\mathrm{K}_{\text {in }}$ channel subunits they form heteromeric channels and modulate the features of $\mathrm{K}_{\text {in }}$ channels.

TPC1 (TPC1a): Two Pore $\mathrm{Ca}^{2+}$-regulated Cation channel 1. A channel-type targeted to the vacuole membrane which activates upon depolarization. TPC1 carries the slow vacuolar SVcurrents and is stimulated by cytosolic $\mathrm{Ca}^{2+}$ and inhibited by luminal $\mathrm{Ca}^{2+}$.

TPC1b: Channels in setaphytes with a TPC1-like structure but with largely modified regions that usually coordinate cytosolic and luminal $\mathrm{Ca}^{2+}$ ions in normal TPC1. 


\section{Box 1: Key evolutionary events in the history of voltage-gated plant $\mathrm{K}^{+}$channels.}

The combination of phylogenetic analyses with structural information and known functional properties allows the identification of four important steps in the evolution of voltage-gated plant Shaker-like $\mathrm{K}^{+}$channels (Figure I, red dotted lines; Figure $\mathbf{2 A}$, red dots): (i) $\mathrm{K}_{\text {in }}$ and $\mathrm{K}_{\text {out }}$ channels share many common structural properties, but were separated already before the split of

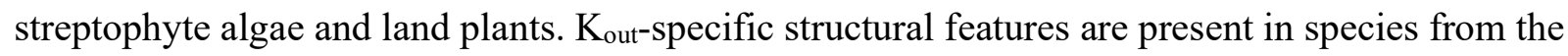
algal class Zygnematophyceae, thought to be sister to land plants, but not in other earlier-diverging streptophyte algae. (ii) In a common ancestor of modern seed plants, the clade of $\mathrm{K}_{\text {weak }}$ channels separated from $K_{\text {in }}$ channels. (iii and iv) In angiosperms, the $K_{\text {in }}$ clade further subdivided into $\mathrm{K}_{\text {silent }}$ (AtKC1-like), $\mathrm{K}_{\mathrm{in}}-\mathrm{a}$ (KAT1-like) and $\mathrm{K}_{\mathrm{in}-\mathrm{b}}$ (AKT1-like) channels. This view is further supported by the observation that members of $K_{i n}-a, K_{i n}-b, K_{\text {silent }}$ and $K_{\text {weak }}$ channel subgroups can form heteromeric $\mathrm{K}^{+}$channels among each other [44,46,73,78-80,104-108]. Likewise, different $\mathrm{K}_{\text {out }}$ channel subunits can co-assemble, but there is no heteromeric assembly between $\mathrm{K}_{\text {in }} / \mathrm{K}_{\text {weak }} / \mathrm{K}_{\text {silent }}$ subunits and $\mathrm{K}_{\text {out }}$ subunits [21].

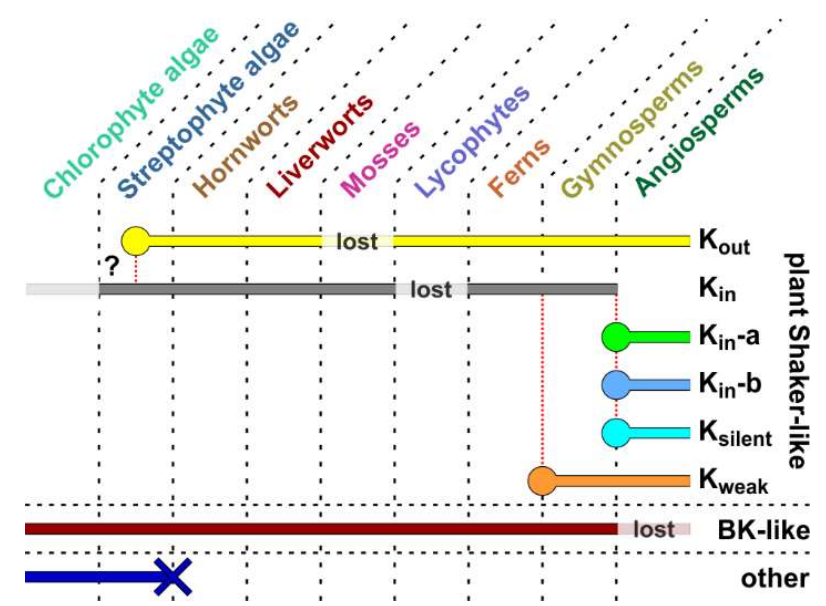

Figure I. Presence of putatively voltage-gated $\mathrm{K}^{+}$channels in plants. While angiosperms have a diverse set of voltage-gated $\mathrm{K}^{+}$channels of the Shaker type $\left(\mathrm{K}_{\mathrm{in}}-\mathrm{a}, \mathrm{K}_{\mathrm{in}}-\mathrm{b}, \mathrm{K}_{\text {silent }}, \mathrm{K}_{\text {weak }}, \mathrm{K}_{\text {out }}\right)$, earlier-diverging plant lines are equipped with different varying sets, reflecting multiple duplication and divergence events during evolution and losses of basic Shaker-types in some lineages $\left(K_{\text {in }}\right.$ in lycophytes and $K_{\text {out }}$ in mosses). These losses might have been partially compensated for by the presence of another type of TPC1-like channels (TPC1b) and/or 
structurally distantly-related BK-like channels $[56,65]$. These channel types are not found in angiosperms. 


\section{Box 2: Characteristic structural patterns of voltage-gated plant $\mathrm{K}^{+}$channels.}

The five known subclasses of GORK-like $\left(\mathrm{K}_{\text {out }}\right)$, AKT2-like $\left(\mathrm{K}_{\text {weak }}\right)$, AtKC1-like $\left(\mathrm{K}_{\text {silent }}\right)$, KAT1like $\left(\mathrm{K}_{\mathrm{in}}-\mathrm{a}\right)$ and AKT1-like $\left(\mathrm{K}_{\mathrm{in}}-\mathrm{b}\right)$ plant $\mathrm{K}^{+}$channels differ in characteristic structural fingerprints (Figure 2B, see also Figures S3, S4, S5, and S6 in the Online Supplemental Information).

- $\mathrm{K}_{\text {out }}$ channels from streptophyte algae to angiosperms have (1) a FTP-motif in the first transmembrane domain S1; (2) a PWDxxYKxxGxxE-motif in a shorter S3-S4-linker; (3) a VELYCTHTAACXFYYLATTxP-motif in S5 together with a 6-aminoacids longer S5-Plinker; (4) a YFAxxTMATxGYGD-motif in the selectivity filter; and (5) a SFDMILGmotif in S6 together with a ALIVKGSxTE-motif at the end of S6 (Figure 2B, yellow; Figure S3).

- $\mathrm{K}_{\text {weak }}$ channels have (1) a glutamine-residue in the S2-S3-linker; (2) a lysine-residue in S4 together with a threonine-residue and a RFS-phosphorylation site in the S4-S5-linker; (3) a methionine-residue in the pore-region; and (4) an RRTMEFRNS-phosphorylation site after S6 (Figure 2B, orange; Figure S4).

- $\mathrm{K}_{\text {silent }}$ channels have (1) an additional amino acid in the S3 segment; (2) a missing positive charge with a [G,C,N]YTY-motif in the pore region; and (3) a FAM-motif after S6 (Figure 2B, cyan; Figure S5). In combination, these motifs can only be found in angiosperms. Nevertheless, a putative channel subunit from $P$. patens shares the peculiar feature of an additional amino acid in S3; and some channel subunits in ferns and bryophytes also lack an otherwise highly conserved arginine-residue in the pore region but have an uncharged amino acid instead.

- $\quad \mathrm{K}_{\mathrm{in}} \mathrm{a}$ (KAT1-like) and $\mathrm{K}_{\mathrm{in}} \mathrm{-b}$ (AKT1-like) channels differ (1) in the S1-S2-linker; (2) in S3; (3) in S4; (4) in the S4-S5 region; and (5) in the cytosolic C-terminus where many KAT1like $\left(\mathrm{K}_{\mathrm{in}}-\mathrm{a}\right)$ channels lack the ankyrin repeat domain that is present in $\mathrm{K}_{\text {in }}-\mathrm{b}$ channels (Figure 2B, green/blue; Figure S6). Despite these differences the two channel types are functionally similar. 


\section{Figures}

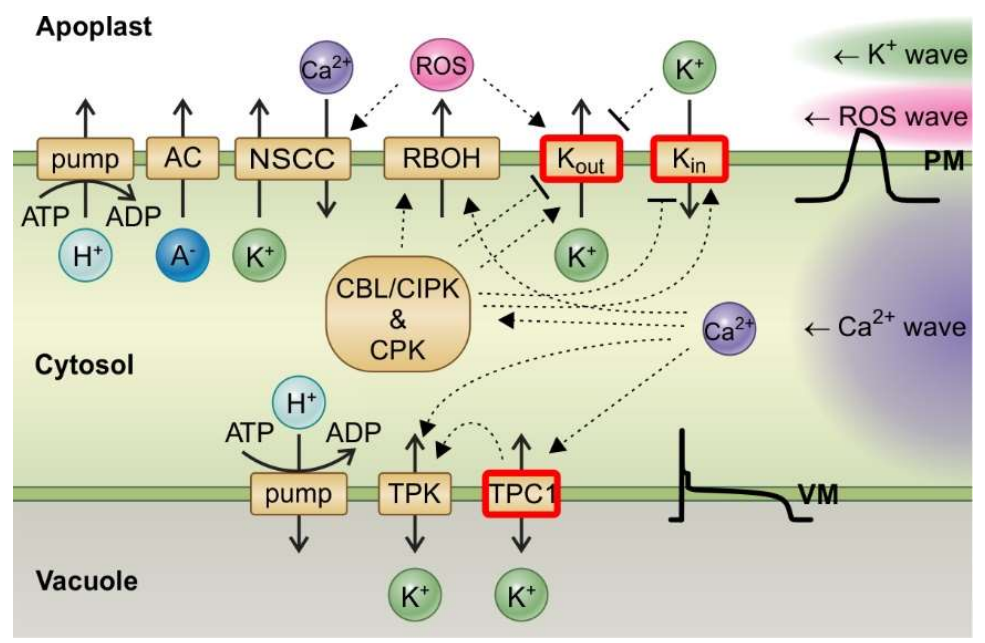

Figure 1. Electric signalling in plants. Electrical signals at the plasma membrane are proposed to involve anion efflux via anion channels (AC), $\mathrm{Ca}^{2+}$ influx via non-selective cation channels (NSCC) and $\mathrm{K}^{+}$efflux and influx via voltage-gated potassium channels ( $\mathrm{K}_{\text {out }}$ and $\left.\mathrm{K}_{\text {in }}\right)$. The entire process is energized by ATP-consuming proton pumps. Electrical signals are accompanied by extracellular waves of potassium and reactive oxygen species (ROS) and by a cytosolic $\mathrm{Ca}^{2+}$ wave [17]. $\mathrm{Ca}^{2+}$ ions affect the activity of membrane proteins (e.g. of the ROS-producing Respiratory Burst Oxidase Homologue, RBOH, family) directly via protein-intrinsic EF-hand motifs and/or indirectly via $\mathrm{Ca}^{2+}$-dependent protein kinases (CBL/CIPK and/or CPK families). The $\mathrm{Ca}^{2+}$ wave is also proposed to be central to vacuolar excitation. This process involves the voltage-gated channel TPC1 together with $\mathrm{K}_{2} \mathrm{P}$-type $\mathrm{K}^{+}$channels (TPK) [16]. 

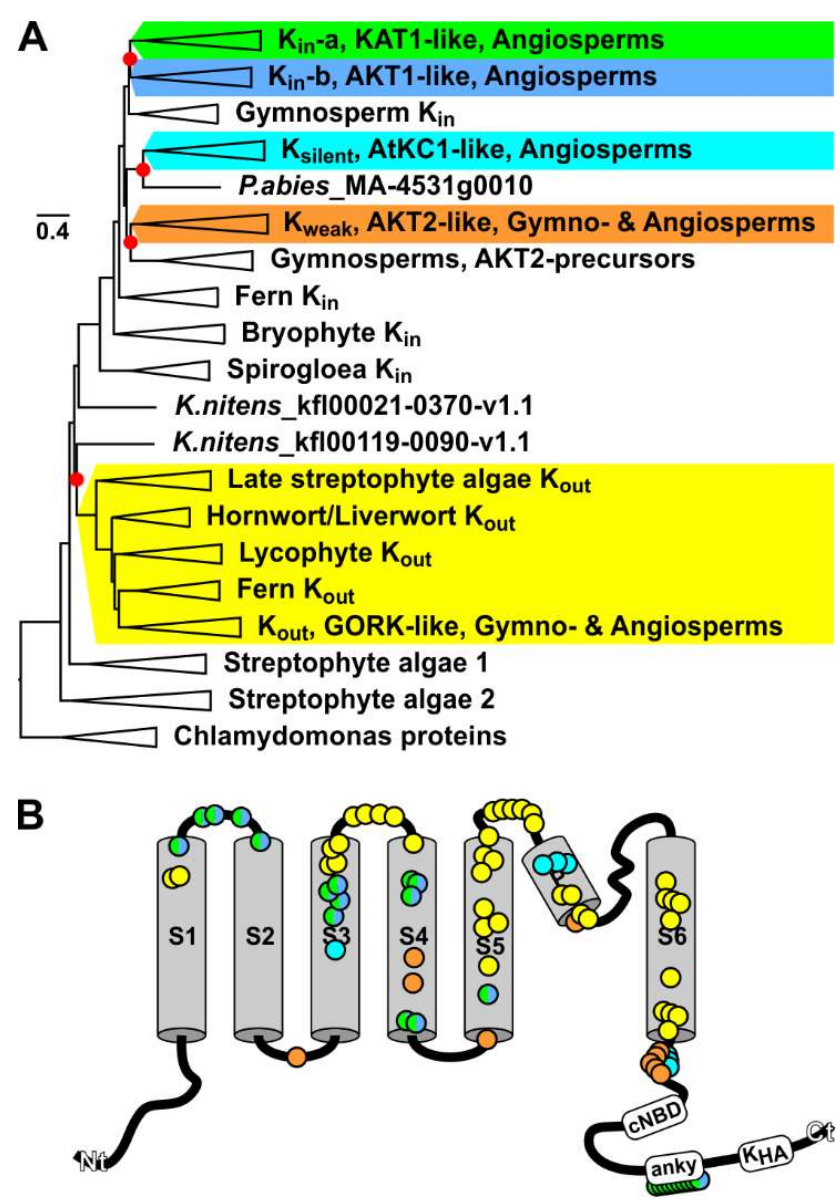

Figure 2. Voltage-gated plant $\mathbf{K}^{+}$channels. (A) Condensed phylogeny of plant Shaker-like channels. The fully expanded tree (see Figure S2 in the Online Supplemental Information) is displayed here with collapsed subclades (triangles). Three proteins (P.abies_MA-4531g0010, K.nitens_kfl00021-0370-v1.1, and K.nitens_kfl00119-0090-v1.1) take on intermediate positions and could not be assigned to any subclade. The five subclades identified in Arabidopsis and confirmed in other angiosperms are coloured: GORK-like $\mathrm{K}_{\text {out }}$ channels (yellow), AKT2-like $\mathrm{K}_{\text {weak }}$ channels (orange), AtKC1-like $\mathrm{K}_{\text {silent }}$ subunits (cyan), KAT1-like $\mathrm{K}_{\text {in-a }}$ channels (green) and AKT1-like $\mathrm{K}_{\text {in }}-\mathrm{b}$ channels (blue). The red dots indicate the branching points that separated each subclade (Box 1) characterized by particular sequence motifs (Box 2). (B) Characteristic fingerprints in plant $\mathrm{K}^{+}$channels. Plant voltage-gated $\mathrm{K}^{+}$channels are composed of four subunits, where a channel subunit is built of six transmembrane regions (S1-S6) and a pore-forming loop and helix (P) between S5 and S6. The cytosolic C-terminal part of a subunit contains a putative cyclic-nucleotide binding-domain (cNBD), might contain an ankyrin-repeat domain (anky) and has at its very end an acidic domain $\left(\mathrm{K}_{\mathrm{HA}}\right)$ involved in channel clustering in the membrane. The 
coloured dots indicate the positions of the fingerprints for GORK-like, $\mathrm{K}_{\text {out }}$ channels (yellow), AKT2-like, $\mathrm{K}_{\text {weak }}$ channels (orange), AtKC1-like, $\mathrm{K}_{\text {silent }}$ channel subunits (cyan) and pinpoint the differences between KAT1-like and AKT1-like $\mathrm{K}_{\text {in }}$ channels (green/blue).
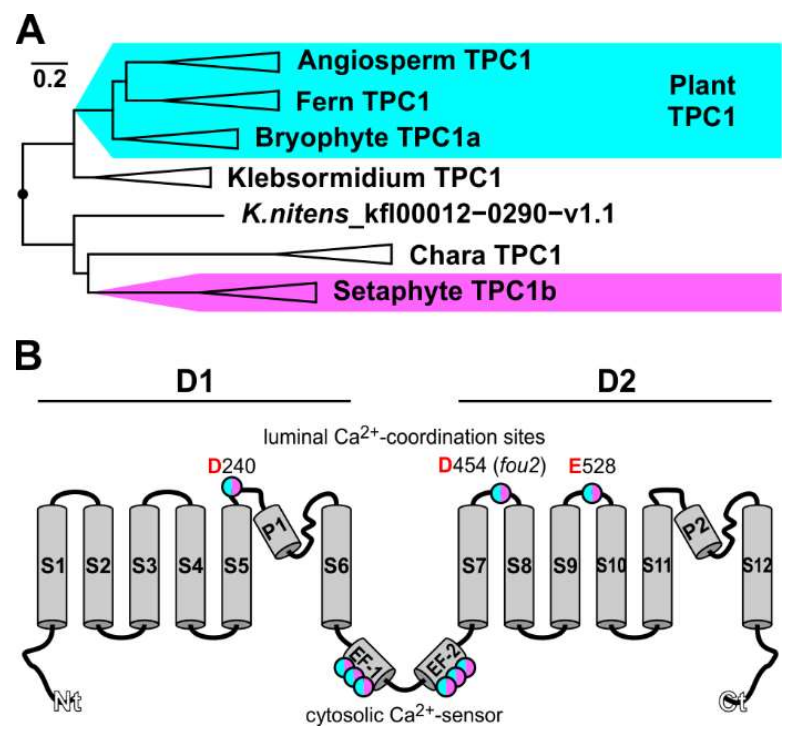

Figure 3. Two-Pore-Cation-Channel 1 (TPC1) in plants. (A) Condensed phylogeny of TPC1. The fully expanded tree (see Figure S7 in the Online Supplemental Information) is displayed here with collapsed subclades (triangles). The protein K.nitens_kf100012-0290-v1.1 takes an intermediate position and could not be assigned to any subclade. The plant TPC1 (cyan) and the Setaphyte TPC1b (magenta) subclades are coloured. (B) Differences in putative $\mathrm{Ca}^{2+}$-binding sites in plant TPCs. Plant TPCs are composed of two subunits, where a channel subunit is built of two Shaker-like modules (D1 and D2, compare with Figure 2B) connected via a cytosolic linker domain. Angiosperm TPCs sense the cytosolic and the luminal $\mathrm{Ca}^{2+}$ concentration. Two EF-hands in the linker domain sense cytosolic $\mathrm{Ca}^{2+}$, while three negatively charged residues (D240, D454, and E538 in the Arabidopsis AtTPC1) coordinate luminal $\mathrm{Ca}^{2+}$. The two subclades TPC1a (or simply TPC1) and TPC $1 \mathrm{~b}$ differ strongly at these $\mathrm{Ca}^{2+}$-sensing sites (coloured dots). 


\section{References}

1 Morris, J.L. et al. (2018) The timescale of early land plant evolution. Proc. Natl. Acad. Sci. U. S. A. 115, E2274-E2283

2 Puttick, M.N. et al. (2018) The Interrelationships of Land Plants and the Nature of the Ancestral Embryophyte. Curr. Biol. 28, 733-745

3 Leebens-Mack, J.H. et al. (2019) One thousand plant transcriptomes and the phylogenomics of green plants. Nature 574, 679-685

4 Harris, B.J. et al. (2020) Phylogenomic Evidence for the Monophyly of Bryophytes and the Reductive Evolution of Stomata. Curr. Biol. 30, 2001-2012

5 Burdon-Sanderson, J.S. (1873) I. Note on the electrical phenomena which accompany irritation of the leaf of Dionæa muscipula. Proc. R. Soc. London 21, 495-496

6 Pfeffer, W. (1873) Physiologische Untersuchungen, Engelmann Verlag.

7 Burdon-Sanderson, J.S. (1874) IV. Note on the excitation of the surface of the cerebral hemispheres by induced currents. Proc. R. Soc. London 22, 368-370

8 Darwin, C.R. (1875) Insectivorous plants, John Murray.

9 Haberlandt, G. (1884) Physiologische Pflanzenanatomie, Engelmann Verlag.

10 Haberlandt, G. (1890) Das reizleitende Gewebesystem der Sinnpflanze, Engelmann Verlag.

11 Beilby, M.J. (2019) Chara braunii genome: a new resource for plant electrophysiology. Biophys. Rev. 11, 235-239

12 Helliwell, K.E. et al. (2019) Alternative Mechanisms for Fast $\mathrm{Na}^{+} / \mathrm{Ca}^{2+}$ Signaling in Eukaryotes via a Novel Class of Single-Domain Voltage-Gated Channels. Curr. Biol. 29, $1503-1511$

13 Hedrich, R. et al. (2016) Electrical Wiring and Long-Distance Plant Communication. Trends Plant Sci. 21, 376-387

14 Cuin, T.A. et al. (2018) The role of potassium channels in Arabidopsis thaliana long distance electrical signalling: AKT2 modulates tissue excitability while GORK shapes action potentials. Int. J. Mol. Sci. 19, 926

15 Drain, A. et al. (2020) Functional characterization and physiological roles of the single Shaker outward $\mathrm{K}^{+}$channel in Medicago truncatula. Plant J. 102, 1249-1265

16 Jaślan, D. et al. (2019) Voltage-dependent gating of SV channel TPC1 confers vacuole excitability. Nat. Commun. 10, 2659

17 Gilroy, S. et al. (2016) ROS, Calcium, and Electric Signals: Key Mediators of Rapid Systemic Signaling in Plants. Plant Physiol. 171, 1606-1615 
18 Pilot, G. et al. (2003) Five-group distribution of the Shaker-like $\mathrm{K}^{+}$channel family in higher plants. J. Mol. Evol. 56, 418-434

19 Sharma, T. et al. (2013) The role of $\mathrm{K}^{+}$channels in uptake and redistribution of potassium in the model plant Arabidopsis thaliana. Front. Plant Sci. 4, 224

20 Anschütz, U. et al. (2014) Going beyond nutrition: Regulation of potassium homoeostasis as a common denominator of plant adaptive responses to environment. J. Plant Physiol. $171,670-687$

21 Dreyer, I. et al. (2004) Assembly of plant Shaker-like $\mathrm{K}_{\text {out }}$ channels requires two distinct sites of the channel $\alpha$-subunit. Biophys. J. 87, 858-872

22 Adem, G.D. et al. (2020) GORK Channel: A Master Switch of Plant Metabolism? Trends Plant Sci. 25, 434-445

23 Peiter, E. et al. (2005) The vacuolar $\mathrm{Ca}^{2+}$-activated channel TPC1 regulates germination and stomatal movement. Nature 434, 404-408

24 Hedrich, R. et al. (2018) Structure and Function of TPC1 Vacuole SV Channel Gains Shape. Mol. Plant 11, 764-775

25 Cheng, S. et al. (2019) Genomes of Subaerial Zygnematophyceae Provide Insights into Land Plant Evolution. Cell 179, 1057-1067

26 Wang, S. et al. (2019) Genomes of early-diverging streptophyte algae shed light on plant terrestrialization. Nat. Plants 6, 95-106

27 Nishiyama, T. et al. (2018) The Chara Genome: Secondary Complexity and Implications for Plant Terrestrialization. Cell 174, 448-464

28 Lang, D. et al. (2018) The Physcomitrella patens chromosome-scale assembly reveals moss genome structure and evolution. Plant J. 93, 515-533

29 Bowman, J.L. et al. (2017) Insights into Land Plant Evolution Garnered from the Marchantia polymorpha Genome. Cell 171, 287-304

30 Pollock, D.D. et al. (2002) Increased Taxon Sampling Is Advantageous for Phylogenetic Inference. Syst. Biol. 51, 664-671

31 Boussau, B. and Scornavacca, C. (2020) Reconciling Gene trees with Species Trees. In Phylogenetics in the Genomic Era (Scornavacca, C. et al., eds), pp. 3.2:1--3.2:23, No commercial publisher $\mid$ Authors open access book

32 Kumar, S. et al. (2017) TimeTree: A Resource for Timelines, Timetrees, and Divergence Times. Mol. Biol. Evol. 34, 1812-1819

33 Hinchliff, C.E. et al. (2015) Synthesis of phylogeny and taxonomy into a comprehensive tree of life. Proc. Natl. Acad. Sci. U. S. A. 112, 12764-12769

34 Dreyer, I. and Blatt, M.R. (2009) What makes a gate? The ins and outs of $\mathrm{K}_{\mathrm{v}}$-like $\mathrm{K}^{+}$ channels in plants. Trends Plant Sci. 14, 383-390

35 Gaymard, F. et al. (1998) Identification and disruption of a plant Shaker-like outward 
channel involved in $\mathrm{K}^{+}$release into the xylem sap. Cell 94, 647-655

36 Ache, P. et al. (2000) GORK, a delayed outward rectifier expressed in guard cells of Arabidopsis thaliana, is a $\mathrm{K}^{+}$-selective, $\mathrm{K}^{+}$-sensing ion channel. FEBS Lett. 486, 93-98

37 Anderson, J.A. et al. (1992) Functional expression of a probable Arabidopsis thaliana potassium channel in Saccharomyces cerevisiae. Proc. Natl. Acad. Sci. U. S. A. 89, 37363740

38 Schachtman, D.P. et al. (1992) Expression of an inward-rectifying potassium channel by the Arabidopsis KAT1 cDNA. Science 258, 1654-1658

39 Pilot, G. et al. (2001) Guard Cell Inward $\mathrm{K}^{+}$Channel Activity in Arabidopsis Involves Expression of the Twin Channel Subunits KAT1 and KAT2. J. Biol. Chem. 276, 32153221

40 Sentenac, H. et al. (1992) Cloning and expression in yeast of a plant potassium ion transport system. Science 256, 663-665

41 Mouline, K. et al. (2002) Pollen tube development and competitive ability are impaired by disruption of a Shaker $\mathrm{K}^{+}$channel in Arabidopsis. Genes Dev. 16, 339-350

42 Marten, I. et al. (1999) AKT3, a phloem-localized $\mathrm{K}^{+}$channel, is blocked by protons. Proc. Natl. Acad. Sci. U. S. A. 96, 7581-7586

43 Lacombe, B. et al. (2000) A Shaker-like $\mathrm{K}^{+}$channel with weak rectification is expressed in both source and sink phloem tissues of Arabidopsis. Plant Cell 12, 837-851

44 Dreyer, I. et al. (1997) Plant $\mathrm{K}^{+}$channel $\alpha$-subunits assemble indiscriminately. Biophys. $J$. $72,2143-2150$

45 Reintanz, B. et al. (2002) AtKC1, a silent Arabidopsis potassium channel $\alpha$-subunit modulates root hair $\mathrm{K}^{+}$influx. Proc. Natl. Acad. Sci. U. S. A. 99, 4079-4084

46 Naso, A. et al. (2006) Stoichiometry studies reveal functional properties of KDC1 in plant Shaker potassium channels. Biophys. J. 91, 3673-3683

47 Duby, G. et al. (2008) AtKC1, a conditionally targeted Shaker-type subunit, regulates the activity of plant $\mathrm{K}^{+}$channels. Plant J. 53, 115-123

48 Amrutha, R.N. et al. (2007) Genome-wide analysis and identification of genes related to potassium transporter families in rice (Oryza sativa L.). Plant Sci. 172, 708-721

49 Villette, J. et al. (2019) Unique features of the grapevine VvK5.1 channel support novel functions for outward $\mathrm{K}^{+}$channels in plants. J. Exp. Bot. 70, 6181-6193

50 Véry, A.-A. et al. (2014) Molecular biology of $\mathrm{K}^{+}$transport across the plant cell membrane: What do we learn from comparison between plant species? J. Plant Physiol. $171,748-769$

51 Chen, G. et al. (2019) Identification of Shaker $\mathrm{K}^{+}$channel family members in Rosaceae and a functional exploration of PbrKAT1. Planta 250, 1911-1925

52 Oikawa, T. et al. (2018) Ion Channels Regulate Nyctinastic Leaf Opening in Samanea 
saman. Curr. Biol. 28, 2230-2238

53 Dreyer, I. et al. (2019) Exploring the fundamental role of potassium channels in novel model plants. J. Exp. Bot. 70, 5985-5989

54 Nguyen, T.H. et al. (2017) A dual role for the OsK5.2 ion channel in stomatal movements and $\mathrm{K}^{+}$loading into xylem sap. Plant Physiol. 174, 2409-2418

55 Long-Tang, H. et al. (2018) Constitutive expression of CmSKOR, an outward $\mathrm{K}^{+}$channel gene from melon, in Arabidopsis thaliana involved in saline tolerance. Plant Sci. 274, 492-502

56 Gomez-Porras, J.L. et al. (2012) Phylogenetic analysis of $\mathrm{K}^{+}$transporters in bryophytes, lycophytes, and flowering plants indicates a specialization of vascular plants. Front. Plant Sci. 3, 167

57 Jegla, T. et al. (2018) Evolution and structural characteristics of plant voltage-gated $\mathrm{K}^{+}$ channels. Plant Cell 30, 2898-2909

58 Riedelsberger, J. et al. (2015) Outward rectification of voltage-gated $\mathrm{K}^{+}$channels evolved at least twice in life history. PLoS One 10, e 0137600

59 Johansson, I. et al. (2006) External $\mathrm{K}^{+}$modulates the activity of the Arabidopsis potassium channel SKOR via an unusual mechanism. Plant J. 46, 269-281

60 Porée, F. et al. (2005) Plant $\mathrm{K}_{\text {in }}$ and $\mathrm{K}_{\text {out }}$ channels: Approaching the trait of opposite rectification by analyzing more than 250 KAT1-SKOR chimeras. Biochem. Biophys. Res. Commun. 332, 465-473

61 Gajdanowicz, P. et al. (2009) Distinct roles of the last transmembrane domain in controlling Arabidopsis $\mathrm{K}^{+}$channel activity. New Phytol. 182, 380-391

62 Riedelsberger, J. et al. (2010) Distributed Structures Underlie Gating Differences between the $\mathrm{K}_{\text {in }}$ Channel KAT1 and the $\mathrm{K}_{\text {out }}$ Channel SKOR. Mol. Plant 3, 236-245

63 Liu, K. et al. (2005) An essential function of phosphatidylinositol phosphates in activation of plant shaker-type $\mathrm{K}^{+}$channels. Plant J. 42, 433-443

64 Li, L. et al. (2008) Single mutations convert an outward $\mathrm{K}^{+}$channel into an inward $\mathrm{K}^{+}$ channel. Proc. Natl. Acad. Sci. U. S. A. 105, 2871-2876

65 Sussmilch, F.C. et al. (2019) On the origins of osmotically driven stomatal movements. New Phytol. 222, 84-90

66 Dreyer, I. et al. (2001) A plant Shaker-like $\mathrm{K}^{+}$channel switches between two distinct gating modes resulting in either inward-rectifying or "leak" current. FEBS Lett. 505, 233239

67 Michard, E. et al. (2005) A unique voltage sensor sensitizes the potassium channel AKT2 to phosphoregulation. J. Gen. Physiol. 126, 605-617

68 Michard, E. et al. (2005) Inward rectification of the AKT2 channel abolished by voltagedependent phosphorylation. Plant J. 44, 783-797 
69 Hoth, S. et al. (2001) The pore of plant $\mathrm{K}^{+}$channels is involved in voltage and ph sensing: Domain-swapping between different $\mathrm{K}^{+}$channel $\alpha$-subunits. Plant Cell 13, 943-952

70 Gajdanowicz, P. et al. (2011) Potassium $\left(\mathrm{K}^{+}\right)$gradients serve as a mobile energy source in plant vascular tissues. Proc. Natl. Acad. Sci. U. S. A. 108, 864-869

71 Sandmann, M. et al. (2011) The $\mathrm{K}^{+}$battery-regulating Arabidopsis $\mathrm{K}^{+}$channel AKT2 is under the control of multiple post-translational steps. Plant Signal. Behav. 6, 558-562

72 Shen, L. et al. (2020) Phosphatidic acid directly binds with rice potassium channel OsAKT2 to inhibit its activity. Plant J. 102, 649-665

73 Ache, P. et al. (2001) VFK1, a Vicia faba $\mathrm{K}^{+}$channel involved in phloem unloading. Plant J. 27, 571-580

74 Philippar, K. et al. (1999) Auxin-induced $\mathrm{K}^{+}$channel expression represents an essential step in coleoptile growth and gravitropism. Proc. Natl. Acad. Sci. U. S. A. 96, 1218612191

75 Nieves-Cordones, M. et al. (2019) Characterization of the grapevine Shaker $\mathrm{K}^{+}$channel VvK3.1 supports its function in massive potassium fluxes necessary for berry potassium loading and pulvinus-actuated leaf movements. New Phytol. 222, 286-300

76 Feng, X. et al. (2020) HvAKT2 and HvHAK1 confer drought tolerance in barley through enhanced leaf mesophyll $\mathrm{H}^{+}$homoeostasis. Plant Biotechnol. J. 18, 1683-1696

77 Geiger, D. et al. (2009) Heteromeric AtKC1·AKT1 channels in Arabidopsis roots facilitate growth under $\mathrm{K}^{+}$-limiting conditions. J. Biol. Chem. 284, 21288-21295

78 Picco, C. et al. (2008) The zinc binding site of the Shaker channel KDC1 from Daucus carota. Biophys. J. 94, 424-433

79 Bregante, M. et al. (2008) KDC1, a carrot Shaker-like potassium channel, reveals its role as a silent regulatory subunit when expressed in plant cells. Plant Mol. Biol. 66, 61-72

80 Naso, A. et al. (2009) The role of the C-terminus for functional heteromerization of the plant channel KDC1. Biophys. J. 96, 4063-4074

$81 \mathrm{Xu}$, J. et al. (2006) A Protein Kinase, Interacting with Two Calcineurin B-like Proteins, Regulates $\mathrm{K}^{+}$Transporter AKT1 in Arabidopsis. Cell 125, 1347-1360

$82 \mathrm{Li}$, L. et al. (2006) $\mathrm{A} \mathrm{Ca}^{2+}$ signaling pathway regulates a $\mathrm{K}^{+}$channel for low-K response in Arabidopsis. Proc. Natl. Acad. Sci. U. S. A. 103, 12625-12630

83 Sánchez-Barrena, M.J. et al. (2020) Recognition and activation of the plant AKT1 potassium channel by the kinase CIPK23. Plant Physiol. 182, 2143-2153

84 Cuéllar, T. et al. (2010) A grapevine Shaker inward $\mathrm{K}^{+}$channel activated by the calcineurin B-like calcium sensor 1-protein kinase CIPK23 network is expressed in grape berries under drought stress conditions. Plant J. 61, 58-69

85 Cuéllar, T. et al. (2013) Potassium transport in developing fleshy fruits: The grapevine inward $\mathrm{K}^{+}$channel VvK1.2 is activated by CIPK-CBL complexes and induced in ripening 
berry flesh cells. Plant J. 73, 1006-1018

86 Kintzer, A.F. and Stroud, R.M. (2018) On the structure and mechanism of two-pore channels. FEBS J. 285, 233-243

87 Hedrich, R. and Neher, E. (1987) Cytoplasmic calcium regulates voltage-dependent ion channels in plant vacuoles. Nature 329, 833-836

88 Kintzer, A.F. and Stroud, R.M. (2016) Structure, inhibition and regulation of two-pore channel TPC1 from Arabidopsis thaliana. Nature 531, 258-262

89 Guo, J. et al. (2016) Structure of the voltage-gated two-pore channel TPC1 from Arabidopsis thaliana. Nature 531, 196-201

90 Schulze, C. et al. (2011) Differential contribution of EF-hands to the $\mathrm{Ca}^{2+}$-dependent activation in the plant two-pore channel TPC1. Plant J. 68, 424-432

91 Pottosin, I. and Dobrovinskaya, O. (2018) Two-pore cation (TPC) channel: Not a shorthanded one. Funct. Plant Biol. 45, 83-92

92 Shabala, S. et al. (2020) The energy cost of the tonoplast futile sodium leak. New Phytol. $225,1105-1110$

93 Rahman, T. et al. (2014) Two-pore channels provide insight into the evolution of voltagegated $\mathrm{Ca}^{2+}$ and $\mathrm{Na}^{+}$channels. Sci. Signal. 7, ra109

94 Patel, S. and Cai, X. (2015) Evolution of acidic $\mathrm{Ca}^{2+}$ stores and their resident $\mathrm{Ca}^{2+}$ permeable channels. Cell Calcium 57, 222-230

95 Kelley, L.A. et al. (2015) The Phyre2 web portal for protein modeling, prediction and analysis. Nat. Protoc. 10, 845-858

96 Bonaventure, G. et al. (2007) A gain-of-function allele of TPC1 activates oxylipin biogenesis after leaf wounding in Arabidopsis. Plant J. 49, 889-898

97 Beyhl, D. et al. (2009) The fou2 mutation in the major vacuolar cation channel TPC1 confers tolerance to inhibitory luminal calcium. Plant J. 58, 715-723

98 Dadacz-Narloch, B. et al. (2011) A novel calcium binding Site in the slow vacuolar cation channel TPC1 senses luminal calcium levels. Plant Cell 23, 2696-2707

99 Jaślan, D. et al. (2016) Gating of the two-pore cation channel AtTPC1 in the plant vacuole is based on a single voltage-sensing domain. Plant Biol. 18, 750-760

100 Cai, S. et al. (2017) Evolutionary conservation of ABA signaling for stomatal closure. Plant Physiol. 174, 732-747

101 Zhao, C. et al. (2019) Evolution of chloroplast retrograde signaling facilitates green plant adaptation to land. Proc. Natl. Acad. Sci. U. S. A. 116, 5015-5020

102 Cai, S. et al. (2017) Speedy Grass Stomata: Emerging Molecular and Evolutionary Features. Mol. Plant 10, 912-914

103 Chen, Z.H. et al. (2017) Molecular Evolution of Grass Stomata. Trends Plant Sci. 22, 
$124-139$

104 Gao, Y.-Q. et al. (2017) The $\mathrm{K}^{+}$channel KZM2 is involved in stomatal movement by modulating inward $\mathrm{K}^{+}$currents in maize guard cells. Plant J. 92, 662-675

105 Lebaudy, A. et al. (2010) Preferential KAT1-KAT2 heteromerization determines inward $\mathrm{K}^{+}$current properties in Arabidopsis guard cells. J. Biol. Chem. 285, 6265-6274

106 Jeanguenin, L. et al. (2008) Heteromerization of Arabidopsis $\mathrm{K}_{\mathrm{v}}$ channel $\alpha$-subunits: Data and prospects. Plant Signal. Behav. 3, 622-625

107 Xicluna, J. et al. (2007) Increased functional diversity of plant $\mathrm{K}^{+}$channels by preferential heteromerization of the Shaker-like subunits AKT2 and KAT2. J. Biol. Chem. 282, 486494

108 Lebaudy, A. et al. (2008) Heteromeric $\mathrm{K}^{+}$channels in plants. Plant J. 54, 1076-1082 